\title{
Reduction of interference using Orthogonal Vertical Beamforming in an indoor communication
}

\author{
Paleerat Wongchampa* \\ Department of Electronics Engineering Technology, College of Industrial Technology, King \\ Mongkut's University of Technology North Bangkok, Thailand
}

\begin{abstract}
The interference between users is one of the factors that limit the achievable user throughput in the current indoor communication. In this paper, an evaluation of transmit beamforming contribution is analyzed in the context of an indoor environment. The technique of the Orthogonal Vertical Beamforming (OVB) has been proposed to reduce interference in an indoor communication. The proposed concept is validated through computer simulation in term of Signal Interference plus Noise Ratio (SINR). The obtained results show that the OVB provides higher performance over the conventional vertical beamforming and Orthogonal Beamforming (OBFM) in an only vertical plane.
\end{abstract}

\section{Introduction}

The wireless communication in indoor mainly used the wireless local area network (WLANs). WLANs has been very popular [1] because a lot of its utilities. WLANs does not only give us the convenience and freedom for the usage but also to set up the WLANs network could possibly connect in house or office computer system. Moreover, there's no need to use uncomfortable signal cable for connecting the network. Portable PC and notebooks could connect freely in the signal-range area to each other or the network from other distances. The IEEE 802.11ac was the new WLANs which would be targeted to replace IEEE 802.11n. The Multiple Input Multiple Output (MIMO) technology has used in IEEE $802.11 \mathrm{ac}$. The MIMO technique needs the feedback information to the system. The beamforming is the technique which without the feedback information. So, the beamforming is less complex than MIMO when using in an indoor communication.

From the work presented in [2], the Orthogonal Beamforming (OBFM) mainly considered the gain provided by the horizontal plane while cannot considering the gain by the vertical plane shown in Fig. 1. The figure 1 (a) and (b) show the top view and side view of the scenario of users stay at the same direction (angle in horizontal plane) but they are located in different distances. In this case, the proposed OBFM cannot handle as it can only the horizontally form one beam to one particular user. The results of conventional vertical beamforming concept [3-4] are low Signal to Interference plus Noise Ratio (SINR) because the interference from other users shown in figure 2 . Therefore, this paper proposes

\footnotetext{
* Corresponding author: paleerat.w@cit.kmutnb.ac.th
} 
the Orthogonal Vertical Beamforming (OVB) to reduce interference in an indoor environment in vertical plane.

The rest of this paper is as follows. After the brief introduction, a brief concept of the OVB is described in Section 2. Then, the computer simulation is performed to show the performance of proposed concept in Section 3. Finally, Section 4 concludes the paper.

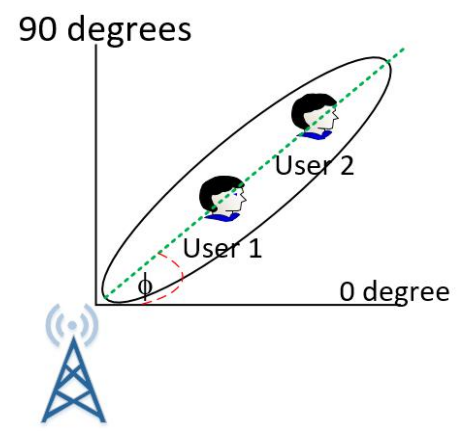

(a)

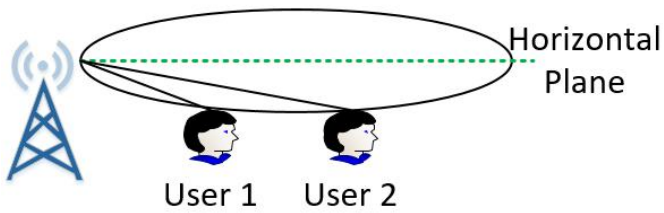

(b)

Fig. 1. Top view (a) and side view (b) in the horizontal beamforming.
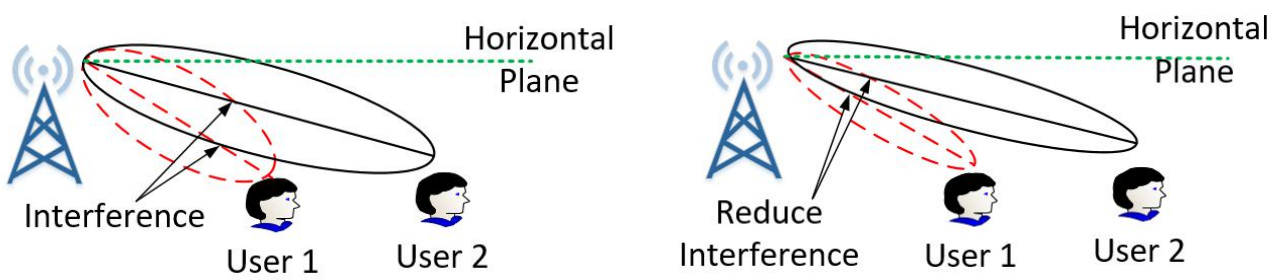

Fig. 2. Conventional vertical beamforming. Fig. 3. Orthogonal vertical beamforming (OVB) concept.

\section{Concept of Orthogonal Vertical Beamforming}

The concept of OVB in this paper is depicted in figure 3. As we can see, the users stay at the same angle in the horizontal plane but they are located at different distances. So, the beam must be able to tilt along with orthogonal in the vertical plane to each user. This paper focuses on a propagation scenario in multi-user communications an indoor environment. Consider a downlink system consisting of an Access Point (AP) consists of $N$ linear array antennas and $M$ users with a single antenna. The vertical antenna pattern relating to AP and the users can be expressed in $\mathrm{dB}$ scale as [5]

$$
A_{V_{M}}=\min \left(12\left(\frac{\theta_{-} \theta_{A P}}{\theta_{3 d B}}\right)^{2}, A_{m}\right)
$$

where $\theta_{3 d B}$ is the vertical $3 \mathrm{~dB}$ beamwidth of the AP antenna. $\theta_{A P}$ is the vertical boresight angles of the AP antenna array. $\theta$ is the vertical angle from AP to the user. $A_{m}$ is the vertical sidelobe attenuation. The characteristic of beam steering for linear array [6] is given in (2).

$$
A F=\sum_{n=1}^{N} e^{j k d_{x} \sin \theta \cos +\beta_{x}}
$$

where $N$ is the number of antenna elements in the $\mathrm{x}$-direction. The antenna elements along the $\mathrm{x}$-axis spaced by $\mathrm{d}_{\mathrm{x}} . \beta_{x}$ is phase delays in antenna elements. $\theta$ and are directions of the direction of arrival in vertical and horizontal plane respectively.

In computer simulation for this paper, considering the scenario where the users are at the same but the different $\theta$. Figure 4 shows the system model for Orthogonal Vertical Beamforming with $M$ users. So, the received signal from the AP can be written as 


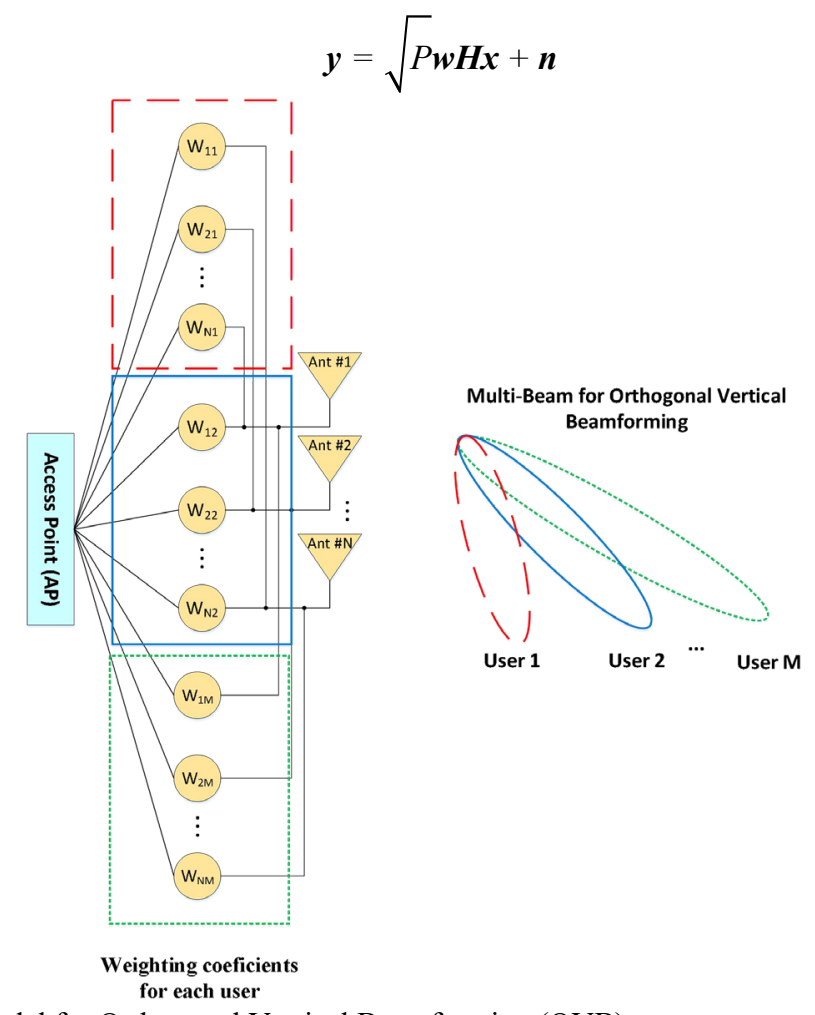

Fig. 4. System model for Orthogonal Vertical Beamforming (OVB).

where $\boldsymbol{y}$ is the received signal vector at AP after performing beam formation and $P$ is the transmitted power of each user, $\boldsymbol{w}$ is weighting coefficient vector of each antenna element at AP, $\boldsymbol{x}$ is the $M \times 1$ signal vector containing the user data $x_{1}, x_{2}, \ldots, x_{m}, \boldsymbol{n}$ is AWGN noise and $\boldsymbol{H}$ is a communication channel for indoor environment include the line of sight, non-line of sight signals and AF of linear array antennas that can be formulated as

$$
\boldsymbol{H}=\boldsymbol{S}_{L O S}+\boldsymbol{S}_{N L O S}
$$

$S_{L O S}$ is the Line-Of-Sight (LOS) signal vector multiplied with the steering vector of the LOS $\left(A F_{L O S}\right)$ signal, $\boldsymbol{S}_{N L O S}$ is the Non-Line-Of-Sight (NLOS) signal vector multiplied with the steering vector of the NLOS $\left(A F_{N L O S}\right)$ signal in equation (2). The system considers in an indoor environment so, each user transmits a signal to the AP that including LOS and NLOS signals. Note that, the power level for both LOS and NLOS components is given by (5) as it is the path loss calculation for indoor environment [6].

$$
\text { path loss }=37-30 \log d+13.8 n^{\left(\frac{n+2}{n+1}-0.46\right)}+X_{\sigma}
$$

where $d$ is the distance between the BS and user, $n$ is the number of floors. Also, $X_{\sigma}$ is zero mean Gaussian distributed random variable (in $\mathrm{dB}$ ) with standard deviation $\sigma$. So, the received signal can be expressed by

$$
\begin{aligned}
& \boldsymbol{y}=\sqrt{P} \boldsymbol{w} \boldsymbol{S}_{L O S} \boldsymbol{x}+\sum_{l=1}^{L} \sqrt{P \boldsymbol{w}} \boldsymbol{S}_{N L O S} \boldsymbol{x}_{\boldsymbol{l}}+\boldsymbol{n} \\
& \boldsymbol{y}=\sqrt{P} \boldsymbol{w}\left(P L_{L O S} \boldsymbol{A} \boldsymbol{F}_{L O S}\right) \boldsymbol{x}+\sum_{l=1}^{L} \sqrt{P} \boldsymbol{w}\left(P L_{N L O S, l} \boldsymbol{A} \boldsymbol{F}_{N L O S,}\right) \boldsymbol{x}_{\boldsymbol{l}}+\boldsymbol{n}
\end{aligned}
$$


where $P L_{L O S}$ is the LOS signal coming from user can be modelled using a simplified path loss. $P L_{N L O S, l}$ is the NLOS signals coming from user and $l$ path can be modelled using Rayleigh fading channel.

The beamforming must be calculate the weighting coefficients for turning the main beam to the users. The weighting coefficients consider in vertical plane. So, assume the users are at the same $\varnothing$ and diferent $\theta$. Then, the $\boldsymbol{w}$ must be inverse of $A F_{L O S}$ signal.

$$
\begin{aligned}
& \boldsymbol{w}=\left(A \boldsymbol{F}_{L O S}\right)^{-1} \\
& \boldsymbol{w}=\left[\begin{array}{llll}
\boldsymbol{A} \boldsymbol{F}\left(\theta_{1}\right) & \boldsymbol{A} \boldsymbol{F}\left(\theta_{2}\right) & \cdots & \boldsymbol{A} \boldsymbol{F}\left(\theta_{M}\right)
\end{array}\right]_{N \times M}{ }^{-1} \\
& \boldsymbol{w}=\left[\begin{array}{cccc}
1 & 1 & \cdots & 1 \\
e^{j k d_{x} \sin \theta_{1} \cos \emptyset+\beta_{x}} & e^{j k d_{x} \sin \theta_{2} \cos \emptyset+\beta_{x}} & \cdots & e^{j k d_{x} \sin \theta_{M} \cos \emptyset+\beta_{x}} \\
\vdots & \cdots & \ddots & \vdots \\
e^{j k d_{x}(N-1) \sin \theta_{1} \cos \emptyset+\beta_{x}} & e^{j k d_{x}(N-1) \sin }{ }_{2} \cos \emptyset+\beta_{x} & \cdots & e^{j k d_{x}(N-1) \sin \theta_{M} \cos \emptyset+\beta_{x}}
\end{array}\right]^{-1}
\end{aligned}
$$

The proposed concept is to find an appropriate weighting coefficients $\boldsymbol{w}$ to avoid interference in the main beam direction as all beams are launched at the same time and the same frequency in the vertical plane. When the system uses the weighting coefficients that the received signal will independent relation between weighting coefficients and steering vectors, so-called orthogonal property. As all beams are orthogonal to one another in the vertical plane, users in the vertical plane are not interference to themselves anymore.

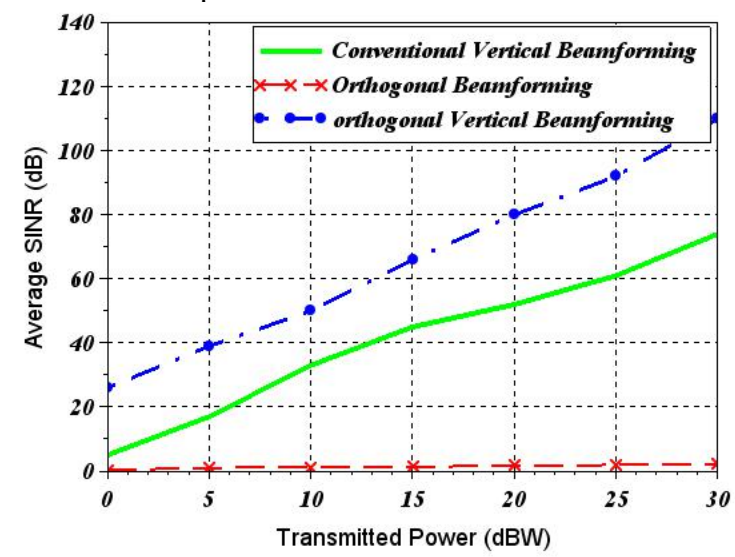

Fig. 5. Average SINR vs. Transmitted power for 4 users that located in vertical angle $(\theta)$ at $20,40,60$ and 80 degrees.

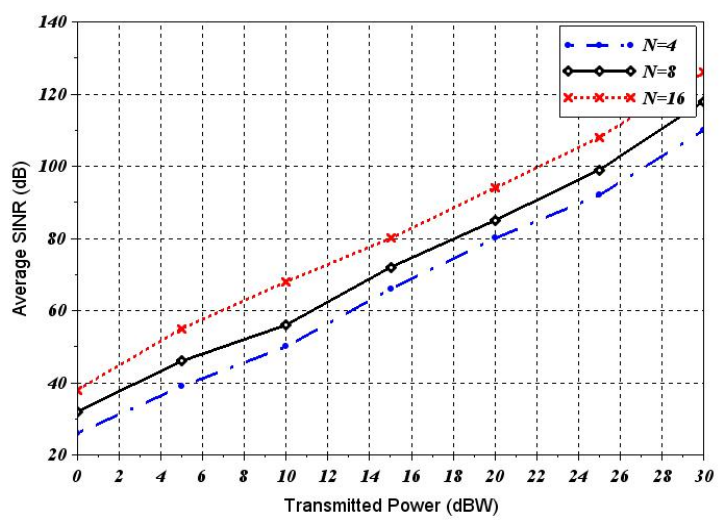

Fig. 6. Average SINR vs. Transmitted power for orthogonal vertical beamforming when $N=4, \mathrm{~N}=8$ and $N=16$. 


\section{Simmulation results}

In this section, we present simulation results to show the performance of proposed Orthogonal Vertical Beamforming (OVB) in indoor communications compared with conventional vertical beamforming and Orthogonal Beamforming (OBFM). We show the impact of the system SINR on the transmitted power. Fig. 5 shows the plots of simulated average SINR vs. transmitted power where the AP is equipped with 4 omnidirectional antennas. The 4-users are located in vertical angle $(\theta)$ at 20, 40, 60 and 80 degrees when all users are an equally horizontal plane $(\varnothing)$ given at 30 degrees. For the simulation, we assume a practical indoor scenario with the path-loss exponent of 1.4. We substitute parameters and vary the transmitted power of user from 0 to $30 \mathrm{dBW}$. Then, the average SINR increases when the transmitted power of user increases.

Moreover, the proposed orthogonal vertical beamforming provides a higher average SINR comparing to conventional vertical beamforming and orthogonal beamforming. The OBFM provides the lowest SINR because of all users at the same horizontal plane $(\varnothing)$. So, the interference cannot be effectively rejected as they are all users stay in the region of the main beam. The result confirms that the proposed orthogonal vertical beamforming can increase the performance in indoor communications in case of the users are in the same horizontal angle but different the vertical angle.

For the transmitted power between average SINR at the number of antennas is 4,8 and 16 elements of OVB technique shown in Fig. 6. At the result of OVB technique simulation at four users, the number of antennas is 4 in blue dash line which has lower average SINR than the number of 8 and 16 elements. The increased number of antennas resulted in an increased gain and narrower beam width. So, the interference between users decreased and SINR increased.

\section{Conclusion}

This paper has proposed an Orthogonal Vertical Beamforming (OVB) concept to reduce interference in an indoor environment for users are in the same horizontal angle. The results of the system from the simulation that provides higher performance in term of SINR over the conventional vertical beamforming and Orthogonal Beamforming (OBFM) in the vertical plane. The future work will combine the OBFM in the horizontal plane with OVB in the vertical plane for improving the performance in term of SINR and capacity in an indoor environment.

\section{Acknowledgment}

This research was funded by College of Industrial Technology, King Mongkut's University of Technology North Bangkok (Grant No Res-CIT0320/2018)

\section{References}

1. Wireless LAN medium access control (MAC) and physical layer specifications, IEEE802.11Std.,Aug.1999.

(PHY)

2. Paleerat Wongchampa and Monthippa Uthansakul, "Orthogonal Beamforming for Multiuser Wireless Communications", IEEE Antennas\&Propagation magazine, 2017, pp. 38-49.

3. Wookbong Lee et al, "Downlink Vertical Beamforming Designs for Multi-User MISO Systems", IEEE conference 2015. 
4. Jiancun Fan et al, "Vertical beamforming with downtilt optimization in downlink cellular networks", IEEE conference 2015.

5. Radiocommunication Sector of International Telecommunication Union, "Report M.2135-1: Guidelines for Evaluation of Radio Interface Technologies for IMTAdvanced," ITU-R M Series, October 2009.

6. ETSI TR 136 931, “LTE; Evolved Universal Terrestrial Radio Access (EUTRA);Radio Frequency (RF) requirements for LTE Pico Node B" 3GPP TR 36.931 version 9.0.0 Release 9. 